\title{
Age Dating Oil and Gas Wastewater Spills Using Radium Isotopes and Their Decay Products in Impacted Soil and Sediment
}

Nancy Lauerł, Avner Vengosh†*

$\dagger$ Division of Earth and Ocean Sciences, Nicholas School of the Environment, Duke

University, Durham, NC 27708, USA

*Corresponding Author: E-mail: vengosh@duke.edu. Phone: 919-681-8050. Fax: 919684-5833.

\section{Contents}

1. Supporting Figures

2. Supporting Tables
Page

$\mathrm{S} 2-\mathrm{S} 3$

S4 


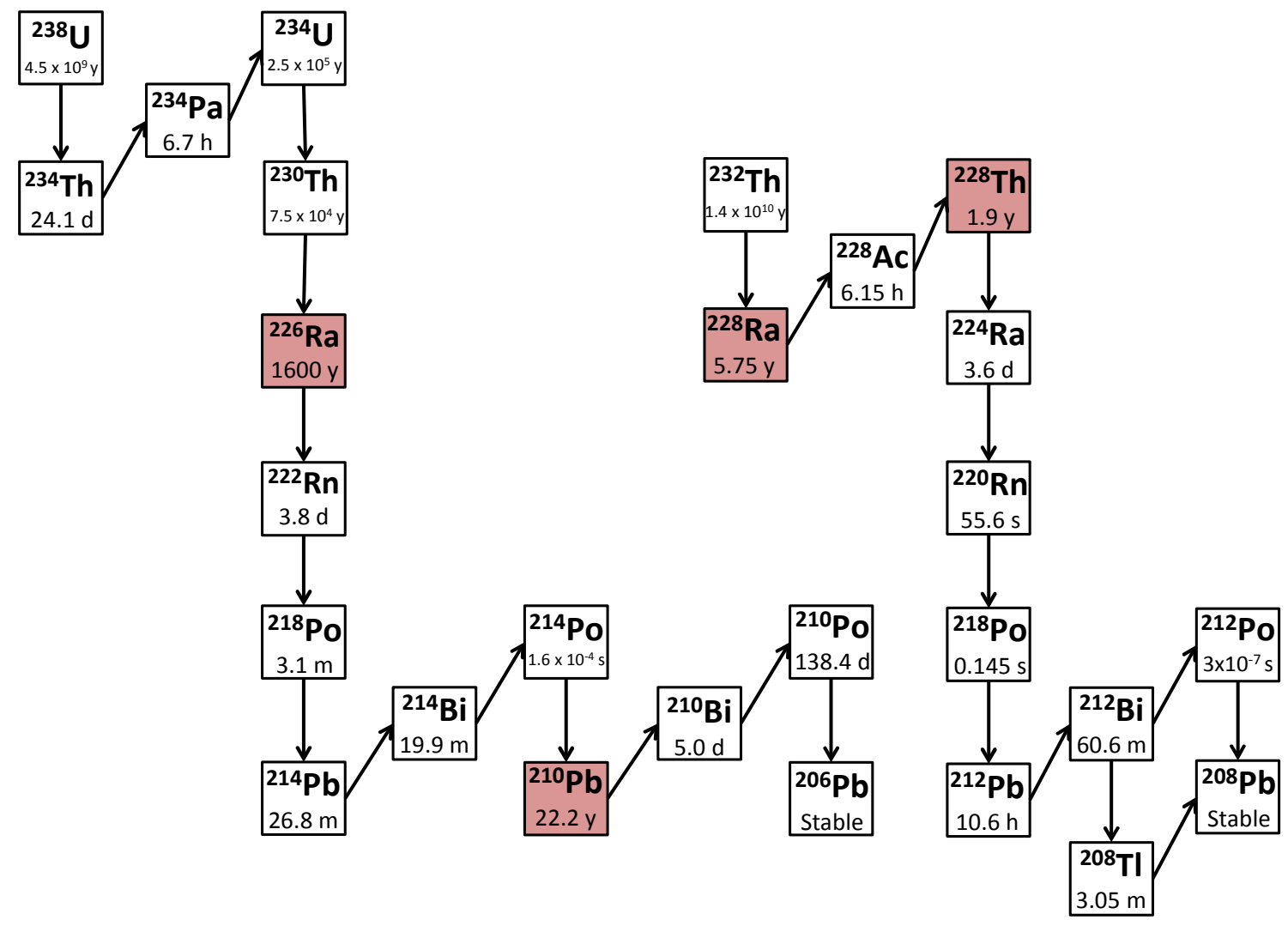

Figure S1. Uranium-Thorium Series. Half-lives for each radionuclide are presented as seconds (s), minutes (m), hours (h), days (d), or years (y) and were acquired from the National Nuclear Data Center (http://www.nndc.bnl.gov/chart/). Downward arrows indicate that the nuclide decays by alpha decay. Upward arrows indicate that the nuclide decays by beta decay. Boxes highlighted in red indicate radionuclides that were the focus of this study. 


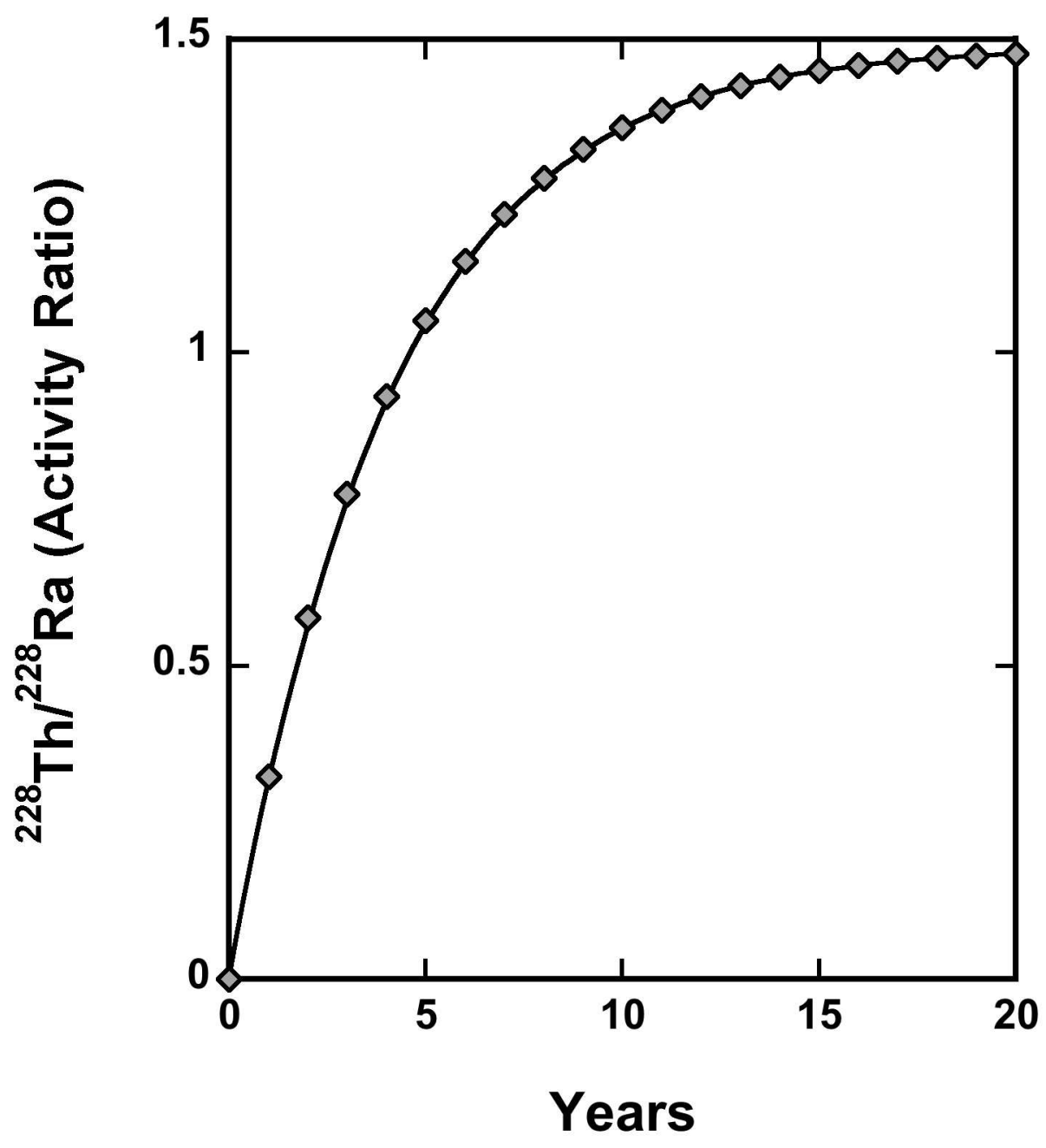

Figure S2. A graph depicting the increase of ${ }^{228} \mathrm{Th} /{ }^{228} \mathrm{Ra}$ (activity ratio) with time due to the decay of ${ }^{228} \mathrm{Ra}$ and the subsequent ingrowth of ${ }^{228} \mathrm{Th}$ in systems where ${ }^{228} \mathrm{Ra}$ is separated from the rest of the thorium decay chain. After approximately 20 years, the ${ }^{228} \mathrm{Th} /{ }^{228} \mathrm{Ra}$ activity ratio will approach $\sim 1.5$. 


\section{Supporting Information}

Table S1. U-Th series radionuclides in soils and sediments impacted by oil and gas wastewater spills.

\begin{tabular}{|c|c|c|c|c|c|c|c|c|c|c|c|}
\hline Spill Name & ${ }^{228} \mathbf{R a}$ & $2 \sigma$ & ${ }^{226} \mathrm{Ra}$ & $2 \sigma$ & ${ }^{210} \mathrm{~Pb}$ & $2 \sigma$ & ${ }^{228} \mathrm{Th}$ & $2 \sigma$ & ${ }^{228} \mathrm{Ra} /{ }^{226} \mathrm{Ra}$ & ${ }^{210} \mathrm{~Pb} /{ }^{226} \mathrm{Ra}$ & ${ }^{228} \mathrm{Th} /{ }^{228} \mathrm{Ra}$ \\
\hline Blacktail Creek 1 & 1883 & $57(3.0 \%)$ & 2802 & $25(0.9 \%)$ & 209 & $28(13.4 \%)$ & 628 & $10(1.6 \%)$ & 0.67 & 0.07 & 0.33 \\
\hline Blacktail Creek 2 & 227 & $8(3.5 \%)$ & 326 & $5(1.5 \%)$ & 63 & $5(7.9 \%)$ & 87 & $2(2.3 \%)$ & 0.70 & 0.19 & 0.38 \\
\hline Blacktail Creek 3 & 647 & $15(2.3 \%)$ & 959 & $6(0.6 \%)$ & 123 & $6(4.9 \%)$ & 247 & $3(1.2 \%)$ & 0.67 & 0.13 & 0.38 \\
\hline Tyler County 1A & 76 & $5(6.6 \%)$ & 267 & $4(1.5 \%)$ & 101 & $8(7.9 \%)$ & 34 & $1(2.9 \%)$ & 0.28 & 0.38 & 0.45 \\
\hline Tyler County 1B & 68 & $7(10.3 \%)$ & 267 & $7(2.6 \%)$ & 105 & $15(14.3 \%)$ & 48 & $2(4.2 \%)$ & 0.25 & 0.39 & 0.71 \\
\hline Tyler County $1 \mathrm{C}$ & 65 & $4(6.2 \%)$ & 266 & $4(1.5 \%)$ & 110 & $10(9.1 \%)$ & 53 & $2(3.8 \%)$ & 0.24 & 0.41 & 0.82 \\
\hline \multicolumn{12}{|l|}{$\begin{array}{l}\text { Background } \\
\underline{\text { Samples }}\end{array}$} \\
\hline Blacktail Creek & 23 & $2(8.7 \%)$ & 22 & $1(4.5 \%)$ & 33 & $4(12.1 \%)$ & 23 & $1(4.3 \%)$ & 1.04 & 1.47 & 0.97 \\
\hline Blacktail Creek & 22 & $1(4.5 \%)$ & 25 & $0.4(1.6 \%)$ & No data & --- & 19 & $0.3(1.6 \%)$ & 0.86 & --- & 0.86 \\
\hline Blacktail Creek & 9 & $2(22.2 \%)$ & 12 & $1(8.3 \%)$ & No data & --- & 11 & $1(9.0 \%)$ & 0.75 & --- & 1.2 \\
\hline Tyler County & 50 & $3(6.0 \%)$ & 51 & $1(2.0 \%)$ & 68 & $5(7.4 \%)$ & 45 & $1(2.2 \%)$ & 0.98 & 1.33 & 0.90 \\
\hline
\end{tabular}

Data and errors are presented as $\mathrm{Bq} / \mathrm{kg}$. Percent errors are also provided in parentheses. Tyler County samples 1A, 1B, and 1C are the sample measured on three different occasions. 